\title{
Orbital self-interaction in Hartree-Fock and density functional theories
}

\author{
John F Dobson $¥ \dagger$ and James H Rose $\uparrow \S$ \\ $\dagger$ Physics Department, University of Michigan, Ann Arbor, Mich, 48109, USA \\ ¥ School of Science, Griffith University, Nathan, Queensland 4111, Australia (permanent \\ address) \\ \& Ames Laboratory, Iowa State University, Ames, Iowa 50011, USA (permanent \\ address)
}

Received 7 July 1982

\begin{abstract}
A synthesis of Hartree-Fock and spin density functional theories yields a fully correlated many-electron energy minimisation method which avoids orbital self-interaction without requiring the use of localised orbitals. The new method is designed primarily for use with extended systems and may facilitate calculation of the band structure of semiconductors and insulators.
\end{abstract}

The rather successful local spin density functional (LSDF) theory of the non-uniform electron gas (Kohn and Sham 1965, Von Barth and Hedin 1972, Gunnarsson and Lundqvist 1976) has an unphysical feature; it permits a localised electron to interact with itself (see Perdew and Zunger 1981 and references therein). Non-local theories (e.g. Gunnarsson et al 1979) partially remove the difficulty, but the most computationally tractable remedy to date has been to subtract the self-interaction of localised orbitals directly, after LDF or LSDF calculations have been performed (Perdew and Zunger 1981). This remedy has the drawback that it requires use of 'the most localised' orbitals, which can be an inconvenient, and even an ill-defined, prescription.

The method which we propose here does not have this drawback, yet it removes self-interaction identically. The essential idea stems from the observation that Hartree-Fock (HF) theory avoids orbital self-interaction completely, because such effects cancel identically between the direct and exchange terms. HF contains no correlation between unlike spins, however, and it incorrectly estimates the manner in which like spins avoid each other. One could imagine formally replacing the Coulomb potential $e^{2} /\left|\boldsymbol{r}-\boldsymbol{r}^{\prime}\right|$ by some effective spin-dependent potential $u\left(r s: \boldsymbol{r}^{\prime} s^{\prime}\right)$ throughout the HF energy expression; this will maintain the cancellation of self-interaction terms. $u$ can be softer than the Coulomb potential at short range, to simulate repulsive correlation, but it must reduce to $e^{2} /\left|\boldsymbol{r}-\boldsymbol{r}^{\prime}\right|$ at long range to maintain charge neutrality. In the following paragraphs we derive a theory of this type. One could also imagine similarly softening the Coulomb interaction in the Hartree-Fock-Schrödinger equation for each spin orbital $\varphi_{i}(r, s)$; we have not, however, found a convincing derivation of this latter type of theory so far. 
Let the true electronic Hamiltonian contain external potential $v(r)$ and external magnetic field $B(\boldsymbol{r})=\boldsymbol{b}(\boldsymbol{r}) / \mu_{\mathrm{B}}$ (where $\mu_{\mathrm{B}}$ is the Bohr magneton), plus the Coulomb pair potential $e^{2} / \boldsymbol{r}-\boldsymbol{r}^{\prime} \mid$. We consider a set of spin orbitals $\left\{\varphi_{i}(r, s)\right\}$ which are the lowest $N$ eigenfunctions of a fictitious one-electron Hamiltonian

$$
\hat{\mathscr{H}}_{1}=\sum_{s s^{\prime}} \int \mathrm{d}^{3} r \hat{\psi}^{\dagger}(\boldsymbol{r}, s)\left[\left(\frac{-\hbar^{2}}{2 m} \nabla^{2}+v_{1}(\boldsymbol{r})\right) \dot{\delta}_{s s^{\prime}}+\boldsymbol{b}_{1}(\boldsymbol{r}) \cdot \boldsymbol{\sigma}_{s s^{\prime}}\right] \hat{\psi}\left(\boldsymbol{r}, \boldsymbol{s}^{\prime}\right) .
$$

In what follows, $v_{1}(r)$ and $b_{1}(r)$ are treated as variational parameters (see Rose and Shore 1976 for a discussion of this strategy in the context of LSDF theory). We construct the trial spin density

$$
\rho\left(\boldsymbol{r}, s, s^{\prime}\right)=\sum_{i=1}^{N} \varphi_{1}^{*}(\boldsymbol{r}, s) \varphi_{i}\left(\boldsymbol{r}, s^{\prime}\right) .
$$

The inhomogeneous energy formula of Gunnarsson and Lundqvist (1976), generalised to include spin, can then be used to provide the following bound for the energy $E$ of the true ground state (assumed non-degenerate):

$$
\begin{gathered}
E \leqslant T_{0}\left[\rho\left(r, s, s^{\prime}\right)\right]+\sum_{s, s^{\prime}} \int\left(\delta_{s s^{\prime}} v(r)+b(r) \cdot \sigma_{s s^{\prime}}\right) \rho\left(r, s, s^{\prime}\right) \mathrm{d}^{3} r \\
+\frac{e^{2}}{2} \sum_{s, s^{\prime}} \int_{0}^{1} \mathrm{~d} \lambda \iint \frac{n_{2 \lambda}\left(\boldsymbol{r}, s: \boldsymbol{r}^{\prime}, s^{\prime}\right)}{\left|\boldsymbol{r}-\boldsymbol{r}^{\prime}\right|} \mathrm{d}^{3} r \mathrm{~d}^{3} r^{\prime} .
\end{gathered}
$$

Here

$$
n_{2 \lambda}\left(\boldsymbol{r}, s: \boldsymbol{r}^{\prime}, s^{\prime}\right)=\left\langle\lambda\left|\hat{\psi}^{\dagger}(\boldsymbol{r}, s) \hat{\psi}^{\dagger}\left(\boldsymbol{r}^{\prime}, s^{\prime}\right) \hat{\psi}\left(\boldsymbol{r}^{\prime}, s^{\prime}\right) \hat{\psi}(\boldsymbol{r}, s)\right| \lambda\right\rangle
$$

is the (normal-ordered) pair distribution in the groundstate $|\lambda\rangle$ of a Hamiltonian containing a reduced Coulomb pair interaction $\lambda e^{2} /\left|r-r^{\prime}\right|$ plus a local spin-dependent potential $w_{s s^{\prime}}(\boldsymbol{r}, \lambda)=v_{1}(\boldsymbol{r}, \lambda) \delta_{s s^{\prime}}+\boldsymbol{b}_{1}(\boldsymbol{r}, \lambda)$. $\boldsymbol{\sigma}_{s s^{\prime}}$ sufficient to maintain the given trial spin density $\rho\left(\boldsymbol{r}, s, s^{\prime}\right)$ at each $\lambda$. (For discussion of the uniqueness of $|\lambda\rangle$ and the nonuniqueness of $w_{s s^{\prime}}$ see Von Barth and Hedin 1972). The Hohenberg-Kohn kinetic energy functional $T_{0}\left[\rho\left(r, s, s^{\prime}\right)\right]$ is the ground-state KE of a non-interacting electron gas with sufficient local potentials to produce the given trial density $\sigma\left(r, s, s^{\prime}\right)$. Since we constructed $\rho$ from the lowest eigenstates $\left\{\varphi_{i}\right\}$ of the trial local one-body Hamiltonian (1), we can immediately write

$$
T_{0}\left[\rho\left(r, s, s^{\prime}\right)\right]=\frac{\hbar^{2}}{2 m} \sum_{i=1}^{N} \sum_{s} \int\left|\nabla \varphi_{i}(r s)\right|^{2} \mathrm{~d}^{3} r .
$$

The usual LSDF theory can be derived (see Gunnarsson et al 1979) by starting with the Hartree expression for $n_{2 \lambda}$ in equation (3), then using an additive exchange-correlation correction based on uniform-gas data. By contrast, we use a Hartree-Fock expression for $n_{2 \lambda}$, corrected by a multiplicative factor to allow for correlation:

$$
\begin{aligned}
n_{2 \lambda}\left(\boldsymbol{r}, s^{\prime} \boldsymbol{r}^{\prime}, s^{\prime}\right) & =n_{2 \lambda}^{\mathrm{HF}}\left(\boldsymbol{r}, s: \boldsymbol{r}^{\prime}, \boldsymbol{s}^{\prime}\right) C_{\lambda}\left(\boldsymbol{r}, s^{\prime}: \boldsymbol{r}^{\prime}, s^{\prime}\right) \\
& \equiv \sum_{i, j=1}^{N} \varphi_{i}^{*}(\boldsymbol{r}, s) \varphi_{j}^{*}\left(\boldsymbol{r}^{\prime}, \boldsymbol{s}^{\prime}\right)\left[\varphi_{j}\left(\boldsymbol{r}^{\prime}, s^{\prime}\right) \varphi_{i}(\boldsymbol{r}, s)-\varphi_{i}\left(\boldsymbol{r}^{\prime}, s^{\prime}\right) \varphi_{j}(\boldsymbol{r}, s)\right] \\
& \times C_{\lambda}\left(\boldsymbol{r}, s: \boldsymbol{r}^{\prime}, \boldsymbol{s}^{\prime}\right) .
\end{aligned}
$$


Our chief approximation consists of replacing the ratio $C_{\lambda}$ by its value in a suitably chosen uniform gas with 'spin-up' and 'spin-down' densities $\bar{\rho}^{ \pm}$:

$C_{\lambda}\left(\boldsymbol{r}, s: \boldsymbol{r}^{\prime}, s^{\prime}\right) \approx n_{2 \lambda}^{\text {unif }}\left(\left|\boldsymbol{r}_{1}-\boldsymbol{r}_{2}\right|, s, s^{\prime}: \bar{\rho}^{+}, \bar{\rho}^{-}\right) / n_{2 \lambda}^{\mathrm{HF} \text { unif }}\left(\boldsymbol{r}_{1}-\boldsymbol{r}_{2} \mid, s, s^{\prime}: \bar{\rho}^{+}, \bar{\rho}^{-}\right)$.

We construct $\bar{\rho}^{ \pm}$from the two eigenvalues $\rho^{+}(\boldsymbol{r}), \rho^{-}(\boldsymbol{r})$ of the $2 \times 2$ trial spin density matrix $\rho\left(\boldsymbol{r}, s, s^{\prime}\right)$ defined in equation (2):

$$
\bar{\rho}^{+}=\frac{1}{2}\left(\rho^{+}(r)+\rho^{+}\left(r^{\prime}\right)\right)
$$

and similarly for $\bar{\rho}^{-}$. Such an average-density ansatz has proved useful elsewhere (see e.g. Gunnarsson et al 1979 and Rose and Dobson 1981).

With the approximation $(7 a),(7 b)$, equation (3) will no longer give a rigorous ground-state energy bound, but in the spirit of density functional theory we should still obtain a useful energy expression:

$$
\begin{aligned}
E \leqslant \sum_{s, s^{\prime}} \sum_{i=1}^{N} \int \varphi_{i}^{*}(\boldsymbol{r}, s)\left[\left(\frac{-\hbar^{2}}{2 m} \nabla^{2}+v(\boldsymbol{r})\right) \delta_{s s^{\prime}}+\boldsymbol{b}(\boldsymbol{r}) \cdot \boldsymbol{\sigma}_{s s^{\prime}}\right] \varphi_{i}\left(\boldsymbol{r}, s^{\prime}\right) \mathrm{d}^{3} \boldsymbol{r} \\
+\frac{1}{2} \sum_{i, j=1}^{N} \int \sum_{s s^{\prime}} \varphi_{i}^{*}(\boldsymbol{r}, s) \varphi_{j}^{*}\left(\boldsymbol{r}^{\prime}, \boldsymbol{s}^{\prime}\right)\left[\varphi_{j}\left(\boldsymbol{r}^{\prime}, \boldsymbol{s}^{\prime}\right) \varphi_{i}(\boldsymbol{r}, s)-\varphi_{i}\left(\boldsymbol{r}^{\prime}, s^{\prime}\right) \varphi_{j}(\boldsymbol{r}, s)\right] \\
\quad \times u\left(\boldsymbol{r}, s: \boldsymbol{r}^{\prime}, \boldsymbol{s}^{\prime}\right)
\end{aligned}
$$

where

$$
u\left(\boldsymbol{r}, s: \boldsymbol{r}^{\prime}, s^{\prime}\right)=\frac{e^{2}}{\left|\boldsymbol{r}-\boldsymbol{r}^{\prime}\right|} \int_{0}^{1} C_{\lambda}\left(\boldsymbol{r}, s ; \boldsymbol{r}^{\prime}, s^{\prime}\right) \mathrm{d} \lambda .
$$

The right-hand side of equation (8) is exactly of Hartree-Fock form, except that the Coulomb pair potential has been replaced by the effective spin-dependent potential $u$ of equation (9). $u$ differs from $e^{2} /\left|\boldsymbol{r}-\boldsymbol{r}^{\prime}\right|$ at short range where the Hartree-Fock and true pair distributions differ significantly. The self-interaction $(i=j)$ terms in equation (8) are manifestly self-cancelling. This arises because the direct and exchange terms in $n_{2}^{\mathrm{HF}}$ were kept together when applying the correlating factor $C_{\lambda}$ in equation (6). This feature is lost if, for example, the exchange is screened, or if the correlation correction is applied additively. We note also that, for full bands, a Wannier transformation from localised to Bloch states preserves the right-hand side of equation (8), for the same reasons as in HF theory. Thus our approach does not depend on the use of localised orbitals.

The simplest way to apply the energy formula (8) is to choose the trial potentials $v_{1}(r), b_{1}(r)$ in equation (1) to be the self-consistent effective potentials from a prior LSDF calculation, so that the $\left\{\varphi_{i}\right\}$ are the LSDF spin orbitals. Equation (8) then gives an improved ground-state energy estimate. Alternatively $v_{1}(\boldsymbol{r})$ and $\boldsymbol{b}_{1}(\boldsymbol{r})$ can be varied independently, giving an even lower approximate energy bound. As in LDF theory, we are only justified in using the formalism derived here for total electronic ground-state energies, but experience with the LDF approach strongly suggests that we interpret the eigenvalues $\varepsilon_{i}$ of the optimised trial one-electron Hamiltonian (1) as band energies. In a similar spirit we might also seek a set of eigenfunctions $\left\{\tilde{\varphi}_{i}\right\}$ and energies $\left\{\tilde{\varepsilon}_{i}\right\}$ of canonical Hartree-Fock equations using our modified potential $u\left(\boldsymbol{r}, s: \boldsymbol{r}^{\prime}, \boldsymbol{s}^{\prime}\right)$ of equation (9) in place of the bare Coulomb potential $e^{2} /\left|\boldsymbol{r}-\boldsymbol{r}^{\prime}\right|$. The $\left\{\tilde{\varphi}_{i}\right\}$ will not, however, belong to a common local one-body Hamiltonian, so it is not obvious that equation (5), and hence equation (8), will hold. 
We should mention that the ansatz (6), (7) does not satisfy the exchange-correlation hole normalisation criterion of Gunnarsson et al (1979) and Gunnarsson and Lundqvist (1976). We have shown, however, that as the number $N$ of electrons approaches infinity, the spurious extra density $\Delta n(r, s)$ obtained by integrating equation (6) is everywhere of $\mathrm{O}(e / V)+\mathrm{O}(n(r, s) / N)$. Hence a total spurious charge of $\mathrm{O}(e)$ is harmlessly spread over the entire volume $V$, with negligible effect on the Coulomb energy per electron. Thus the method should work well for crystals. For small systems one might contemplate an explicit hole-normalisation factor in equation (6), but it is not clear that this will lead to a consistent theory.

Our work is somewhat complementary to that of Stoll et al (1980), who discussed self-interaction but used unmodified HF theory for like spins in small systems. We should also mention two papers which do not discuss self-interaction, but which do introduce a correlating factor via arguments of the Jastrow type. Colle and Salvetti (1975) fit their correlating factor to atomic rather than electron gas data, while Berrondo and Goscinski (1981) discuss the uniform gas but do not appear to account for kinetic correlation energy.

In summary, we have given an energy scheme (equations (8), (9) and (7)) which:

(i) includes both potential and kinetic correlation energy (via a Feynman integration) for electrons of either spin, using local spin density ideas;

(ii) avoids self-interaction in an HF-like manner, which does not rely on the use of localised orbitals;

(iii) should work well for large systems, possibly also giving improved insulator and semiconductor band structures.

It remains to choose an adequate existing uniform-gas pair function $n_{2 \lambda}^{\text {unif }}$ from the literature, and to try some band calculations. Velocity-dependent correlating factors are also under consideration.

We would like to thank Professor L Sander, Dr P. Jewsbury, Dr S Nordholm and Professor J Perdew for helpful discussions. J F Dobson would like to thank the staff of the Physics Department, University of Michigan, for hospitality during January-June 1980, where some of this work was performed. The work at Michigan was supported in part by NSF grant DMR-78-25012. Ames Laboratory is operated by the US Department of Energy by Iowa State University under Contract No. W-7405-ENG-82. J H Rose's work there was supported by the Director for Energy Research, Office of Basic Sciences.

\section{References}

Berrondo M and Goscinski O 1981 Chem. Phys. Lett. 81 75-8

Colle R and Salvetti O 1975 Theor. Chimica Acta 37 329-34

Gunnarsson O, Jonson M and Lundqvist B I 1979 Phys. Rev. B 20 3136-64

Gunnarsson O and Lundquist B I 1976 Phys. Rev. B 13 4274-98

Kohn W and Sham L J 1965 Phys. Rev. 140 A1133-8

Perdew J P and Zunger A 1981 Phys. Rev. B 23 5048-79

Rose J H and Dobson J F 1981 Solid State Commun. 37 91-6

Rose J H and Shore S 1975 Solid State Commun. 17 327-30

Stoll H, Golka E and Preuss H 1980 Theor. Chimica Acta 55 29-41

Von Barth U and Hedin L 1972 J. Phys. C: Solid State Phys. $51629-42$ 